\title{
2. Einleitung
}

2.1. Herz-Kreislaufstillstand 6

2.2. Kardiopulmonale Reanimation 6

$\begin{array}{ll}\text { 2.3. Unterer Ösophagussphinkterdruck und Beatmungsproblematik } & 7\end{array}$

2.4. Reanimationssituation in der zahnärztlichen Privatpraxis 8

2.5. Fragestellung 9

\section{Material und Methode}

$\begin{array}{ll}\text { 3.1. Probanden } & 11\end{array}$

$\begin{array}{ll}\text { 3.2. Testsysteme } & 11\end{array}$

$\begin{array}{ll}\text { 3.3. Versuchsaufbau } & 15\end{array}$

$\begin{array}{ll}\text { 3.4. Methode } & 18\end{array}$

\section{Resultate}

$\begin{array}{lr}\text { 4.1. Tabellarische Darstellung der Testergebnisse } & 19\end{array}$

4.1.1. Lungenvolumina bei hoher Lungencompliance 19

$\begin{array}{ll}\text { 4.1.2. Lungenvolumina bei tiefer Lungencompliance } & 20\end{array}$

$\begin{array}{ll}\text { 4.1.3. Magenvolumina bei hoher Lungencompliance } & 21\end{array}$

4.1.4. Magenvolumina bei tiefer Lungencompliance 22

4.2. Grafische Darstellung der Testergebnisse 23

$\begin{array}{ll}\text { 4.3. Statistik } & 24\end{array}$

$\begin{array}{ll}\text { 4.3.1. Lungenvolumina } & 24\end{array}$

4.3.2. Magenvolumina 25 


\section{Diskussion}

5.1. Lungenvolumina

5.2. Magenblähung

5.3. Vergleich $S B$ und EG/R

5.4. Material und Methode

5.5. Schlussfolgerungen

6. Literaturverzeichnis

7. Verdankungen

8. Curriculum vitae 


\section{Zusammenfassung}

\section{Einleitung}

Bei einem Herzkreislaufstillstand kommt es nach kurzer Zeit zu einem starken Tonusverlust des unteren Ösophagussphinkters. Dies kann dazu führen, dass bei der Beatmung durch ungeschulte oder nur sporadisch geschulte Personen im Rahmen der kardiopulmonalen Reanimation eine Magenblähung auftreten kann. Durch die Stresssituation wird oft zu schnell und/oder zu stark beatmet und schwere Komplikationen wie Aspiration, Pneumonie oder sogar Tod können die Folge davon sein. Um das Risiko einer Magenblähung zu minimieren wurde der SMART BAG®, ein spitzenfluss- und druckbegrenzter Einwegatembeutel entwickelt. Das Ziel dieser Arbeit war, diesen speziellen Beatmungsbeutel mit zwei weiteren Testvorrichtungen in Bezug auf die Grösse der übermittelbaren Tidalvolumina und der auftretenden Magenblähung zu vergleichen.

\section{Material und Methode}

Es wurden drei Einwegatembeutel miteinander verglichen: ein Easy Grip® Einwegatembeutel, ein SMART BAG® und ein Easy Grip® Einwegatembeutel mit vorgeschaltetem 0.5I Rüsch Atembeutel (Reservoir).

14 Zahnärzte kurz nach Staatsexamen am Zentrum für Zahnmedizin der Universität Zürich führten 12 Beatmungszyklen mit je 10 Beatmungen an einem Mini Ventilation Training Analyzer durch. Dabei wurden nach jedem Zyklus der Beatmungsbeutel, die Lungencompliance und/oder der untere Ösophagussphinkterdruck in randomisierter Art verändert. Die erreichten Tidalvolumina und die auftretende Magenblähung konnten anhand von zwei Volumeter gemessen werden. Zur späteren Auswertung wurden die Versuchsdurchführungen mit Hilfe einer Videokamera aufgenommen.

\section{Resultate}

Für jede Versuchsanordnung und jeden Probanden wurden die Mediane der erzielten Tidal- bzw. der verursachten Magenvolumina ausgerechnet.

Mit allen drei Testvorrichtungen konnte unabhängig der Lungencompliance und des unteren Ösophagussphinkterdrucks ein suffizientes Lungenvolumen erreicht werden. Es gab keine statistisch signifikanten Unterschiede zwischen dem SMART BAG® und dem Easy Grip® mit vorgeschaltetem 
Reservoir. Im Vergleich zu den anderen beiden Vorrichtungen konnten jedoch mit dem Easy Grip® signifikant grössere Tidalvolumina erzielt werden.

Eine Magenblähung konnte mit Hilfe des SMART BAG® und des Easy Grip® mit Reservoir nur bei einem unteren Ösophagussphinkterdruck von $1.5 \mathrm{kPa}$ vollständig vermieden werden. Die Lungencompliance spielte dabei keine Rolle. Bei Verwendung des Easy Grip® ohne Reservoir kam es immer zu einem Einströmen von Luft in den Magen.

\section{Diskussion}

Sogar im schlimmsten Fall der Fälle, bei reduziertem Druck des unteren Ösophagussphinkters und verminderter Lungencompliance konnten sowohl mit dem SMART BAG® als auch dem Easy Grip® mit Reservoir suffiziente Atemzugsvolumen erreicht werden. Dabei kam es zu einer signifikant kleineren Magenblähung im Vergleich zum Easy Grip® ohne Reservoir. Bei einer Erhöhung des unteren Ösophagussphinkterdrucks auf $1.5 \mathrm{kPa}$ konnte eine Magenblähung bei diesen beiden Vorrichtungen sogar vollkommen ausgeschlossen werden.

Die Kombination eines standardisierten Beatmungsbeutel mit einem vorgeschaltetem Atembeutel bietet möglicherweise den gleichen Schutz vor einer Magenblähung wie der SMART BAG®. 


\section{Einleitung}

\subsection{Herz-Kreislaufstillstand}

Ein so genannter Herz-Kreislaufstillstand ist ein abrupter Halt der Atembewegung oder eines effizienten Kreislaufes 1. 1. 80\% der Fälle sind tachysystolische Herzstillstände (Kammerflimmern oder -flattern), 20\% asystolische Herzstillstände (Asystolie). Die drei Kardinalsymptome umfassen Bewusstlosigkeit (nach ca. 10-15s), Atemstillstand (nach 30-60s) und Kreislaufstillstand. Die Prodromalsymptome sind oftmals unspezifisch. Brustschmerzen aufgrund von Ischämie, Herzklopfen bei Tachyarrhythmie und Dyspnoe bei Herzinsuffizienz gelten daher als zweideutige Zeichen (2).

Der plötzliche Herzstillstand ist die Haupttodesursache in Europa und betrifft jährlich je nach Definition zwischen 350'000-700'000 Personen (3-5). Auch in den Vereinigten Staaten stellt das akute Herzversagen ein bedeutendes Problem des Gesundheitswesens dar und ist verantwortlich für 300‘000-400‘000 Todesfälle pro Jahr 2,6).

In der Schweiz hat die Inzidenz des plötzlichen Herztodes in den letzten Jahren abgenommen und beträgt 0,4 bis $1 / 1000$ Personen/Jahr 4, 77. Somit sind hierzulande jährlich etwa $8000-10000$ Menschen betroffen 8). Über $80 \%$ dieser Patienten befinden sich in den ersten Minuten nach dem Herzkreislaufstillstand im Kammerflimmern, welches durch frühe Defibrillation mit gutem Resultat behandelt werden kann 9). Die Empfehlung lautet, die Defibrillation innerhalb von maximal acht, am besten innerhalb von fünf Minuten durchzuführen 10 . Die Erfolgschance sinkt beim beobachteten Kreislaufstillstand mit Kammerflimmern ohne kardiopulmonale Reanimation (CPR) jede Minute um $10 \% 11,12$. Trotz grosser Fortschritte in medizinischen Rettungssystemen können nur 3-10\% von Patienten, welche ausserhalb eines Spitals einen Herzkreislaufstillstand erleiden, erfolgreich wiederbelebt werden 6).

Die Risikofaktoren für akutes Herzversagen sind vielfältig. Die Inzidenz steigt mit zunehmendem Alter an, wobei $75 \%$ der Fälle bei Männern auftreten mit einem jährlichen Vorkommen welches 3-4 mal grösser ist als bei Frauen 2.

\subsection{Kardiopulmonale Reanimation}

Da das zentrale Nervensystem bereits nach 3-4 Minuten ohne Sauerstoff irreparable Schäden aufweisen kann, muss sofort nach der Diagnose eines Herz-Kreislaufstillstandes mit den Wiederbelebungsmassnahmen begonnen werden. Zu den Basismassnahmen der kardiopulmonalen 
Reanimation zählen die Herzdruckmassage und die Beatmung. Bei einem Patienten mit ungeschützten Atemwegen wird heute ein Verhältnis von 30 Herzkompressionen zu 2 Beatmungen (30:2) empfohlen, das heisst, dass nach 30 Herzdruckmassagen 2 Beatmungsstösse folgen (13). Bis 2005 betrug dieses Verhältnis noch 15:2. In experimentellen Versuchen konnte jedoch gezeigt werden, dass eine Unterbrechung nach 15 Kompressionen zwecks Beatmung mit einem signifikanten Abfall des koronaren Durchblutungsdruckes (14) einherging. Beim Menschen erzeugt eine Unterbrechung der Herzdruckmassage eine verringerte Wahrscheinlichkeit eines Defibrillationserfolges (15). Idealerweise sollte somit die Herdruckmassage gar nicht unterbrochen werden, wodurch das Leben des Patienten durch mangelnde Beatmung jedoch ebenso gefährdet wird. In Tiermodellen konnte schlussendlich gezeigt werden, dass sich durch ein Verhältnis von 30:2 ein spontaner Kreislauf in einer signifikant kürzeren Erholungszeit einstellen konnte und eine grössere systemische und zerebrale Sauerstoffversorgung gegeben ist 16). Somit wurde 2005 dieses Verhältnis von 15:2 auf 30:2 angehoben (13).

\subsection{Unterer Ösophagussphinkter und Beatmungsproblematik}

Am Übergang vom Ösophagus in den Magen befindet sich eine Zone mit erhöhtem Druck, welche unterer Ösophagussphinkter genannt wird 17). Dabei handelt es sich nicht um einen echten, sondern vielmehr um einen funktionellen Schliessmuskel. Die Tunica muscularis der Speiseröhre besteht aus einer inneren Ring- und einer äusseren Längsmuskelschicht. Die Längsfaserschicht und die schraubenförmig nach innen abstrahlenden Fasern der Zirkulärschicht sind so gegen die Längsachse des Rohres verdreht, dass sie gemeinsam mit einem in der Propria gelegenen Venenplexus einen sogenannten angiomuskulären Dehnverschluss bilden. Ist die Speiseröhre gespannt und lang, so ist sie dicht (18). Da der Ösophagus im Ruhezustand unter einer gewissen Längsspannung steht, kommt es somit ohne die Ausbildung eines echten Sphinkters zu einem dichten Verschluss des Ösophagus gegen den Magen, wodurch ein Reflux des Mageninhaltes verhindert wird.

Die Verteilung des Beatmungsvolumens in einem Patienten mit ungeschützten Atemwegen hängt u.a. vom Druck des unteren Ösophagussphinkters (UÖSD), vom Widerstand in den Atemwegen und von der Lungencompliance ab (19). Bei einem Herzstillstand kommt es zu einem schnellen Druckabfall des UÖSD von ca. $2 \mathrm{kPa}\left(20 \mathrm{~cm} \mathrm{H}_{2} \mathrm{O}\right)$ zu ca. 0.5kPa $\left(5 \mathrm{~cm} \mathrm{H}_{2} \mathrm{O}\right)$ 20-22). Durch den Druckabfall kann während der Beatmungsphase vermehrt Luft in den Magen eintreten und es kommt zu einer Magenblähung. Diese erhöht den intragastrialen Druck (23), hebt das Zwerchfell an, beschränkt die 
Lungenbewegung und setzt die Lungencompliance und somit auch die Ventilation weiter herab 24 , 25). Dadurch können schwere Komplikationen wie Aspiration, Pneumonie oder sogar Tod eintreten $26,27)$

\subsection{Reanimationssituation in der zahnärztlichen Privatpraxis}

Kleinere medizinische Notfälle können in jeder Zahnarztpraxis vorkommen. Dazu zählen zum Beispiel die vasovagale Synkope, Asthmaanfälle, Anaphylaxie, epileptische Anfälle oder auch das Verschlucken und Aspirieren von Fremdkörpern. N.M. Girdler und D.G. Smith haben in einer Studie untersucht, wie häufig solche Notfälle in britischen Praxen vorkommen und wie gut die Zahnärzte geschult sind. Über einen Zeitraum von 12 Monaten erlitten Patienten am häufigsten eine vasovagale Synkope (596 Patienten), gefolgt von Hypoglykämie (54 Patienten), Angina pectoris (53 Patienten) und epileptischen Anfällen (42 Patienten). Weniger häufig traten Asthma- oder Erstickungsanfälle auf, nur selten sogar Anaphylaxie, hypertensive Krise und Myokardinfarkt. Ein Herzkreislaufstillstand kam bei nur einem Patienten vor (28).

Einen ersten schriftlichen Bericht über einen Herzkreislaufstillstand mit erfolgreicher Wiederbelebung in einer zahnärztlichen Praxis lieferte P. L. Hunter. Der Autor gibt in seinen damaligen Ausführungen an, dass wiederholte und regelmässige Schulung in kardiopulmonaler Reanimation eine grosse Rolle spielen, um eine solche Stresssituation bewältigen zu können 29). Bereits zwei Jahre zuvor berichtete Brahms über den Tod von zwei Kindern in Folge einer Anästhesie mit Methohexiton respektive Halothan. Dies lenkte den Fokus ebenso auf die Notwendigkeit, dass auch zahnärztliches Personal in CPR geschult sein muss (30).

In einer deutschen Studie, welche 2008 publiziert wurde, ermittelte man mit Hilfe von Fragebogen sämtliche Notfälle, welche in einer Zeitspanne von 12 Monaten bei Zahnärzten aus dem Bundesland Sachsen-Anhalt auftraten. 620 Zahnärzte von 2998 nahmen an der Studie teil. Davon meldeten 57\% der Zahnärzte bis zu 3 Notfälle und 36\% der Zahnärzte bis zu 10 Notfälle während diesem Zeitraum. Wie bei der Studie von Girdler und Smith stellte die vasovagale Synkope den häufigsten Zwischenfall dar (1238 Fälle). Da zwei Herzkreislaufstillstände auftraten, rechnete man das Vorkommen dieses schweren Notfalles in einer Zahnarztpraxis in Sachsen-Anhalt auf 1:638960 Patienten (31).

Bei einer ähnlichen Studie, welche in Australien durchgeführt worden war, nahmen von ursprünglich 350 Zahnärzten 45\% teil (157 Personen). Dabei zeigte sich, dass über 5 Prozent dieser Zahnärzte 
bereits einen Patienten reanimieren mussten. 2/3 der Teilnehmer fühlten sich fähig, eine kardiopulmonale Reanimation durchführen zu können 32

Trotz des relativ seltenen Vorkommens eines Herzkreislaufstillstandes in einer zahnärztlichen Praxis ist es wichtig, dass Zahnärzte neben kleineren eben auch mit diesen lebensbedrohenden Notfällen umgehen können. So sollten sie also auch genügend in kardiopulmonaler Reanimation geschult werden. Leider sind auch heute noch viele Gesundheitsbedienstete unfähig, lebensrettende Sofortmassnahmen zu ergreifen und es wird nicht genug Aufwand betrieben, um für solche Situationen geschult zu werden 33,34 .

Eine Umfrage bei 199 Zahnärzten in Neuseeland zeigte, dass in den letzten 10 Jahren in 129 Praxen (65.2\%) medizinische Notfälle aufgetreten waren mit durchschnittlich 2 Vorfällen pro 10000 Patienten. Auch hier war die vasovagale Synkope ein häufiger Zwischenfall, welcher in 121 Praxen (61.1\%) mit einer durchschnittlichen Häufigkeit von 6,9 pro 10000 Patienten auftrat. Die interessanteste Aussage war jedoch, dass mehr als die Hälfte der Zahnärzte unzufrieden war mit der Schulung in CPR, welche sie als Studenten erhalten hatten. 28 (14.1\%) fühlten sich zum Zeitpunkt der Befragung nur mangelhaft vorbereitet für einen eventuell auftretenden Notfall 35.).

An der Universität Zürich werden die Studenten der Zahnmedizin momentan 4 Stunden im 3. Studienjahr und 4 Stunden in 5. Studienjahr in Reanimation geschult (Stand Januar 2012).

\subsection{Fragestellung}

Eine kardiopulmonale Reanimation stellt für ungeschulte oder nur sporadisch geschulte Personen, zu welchen man das zahnärztliche Personal einbeziehen muss, eine Stresssituation dar. Durch den dabei erhöhten Beatmungsdruck kann es somit zu einer Magenblähung und den bereits aufgeführten Komplikationen kommen.

Der in der Zahnarztpraxis am häufigsten verwendete Beatmungsbeutel bei kardiopulmonalen Reanimationssituationen ist der Easy Grip (EG). Durch starkes Zusammendrücken kann mit diesem Beutel ein grosses Volumen produziert werden. Gleichzeitig kann aber der Atemwegsspitzendruck den UÖSD überschreiten und die überschüssige Menge an Luft gelangt in den Magen. Das Problem der Magenblähung ist also bei Verwendung des EG nur schwer kontrollierbar.

O-TWO Medical Technologies Inc produzieren einen speziellen Beatmungsbeutel, den SMART BAG® (SB). SMART steht für Synchronized Manual Actuation Response Technology. Bei zu starkem Zusammenpressen des Beutels limitiert ein druckgegensteuerndes Ventil den inspiratorischen 
Gasfluss und damit den Atemwegsspitzendruck bis zu maximal 19 mbar (1.9 kPa). Das Risiko der Magenblähung kann somit vermindert werden.

Ein ähnliches Resultat kann mit einem dem EG vorgeschalteten Reservoir (EG/R) erzeugt werden (36). Bei zu starkem Druck wird die überflüssige Luft in das Reservoir abgegeben und kann anschliessend langsam abfliessen. Der Atemwegsspitzendruck wird ebenfalls vermindert.

Das Ziel unserer Studie war, diese drei Vorrichtungen (EG, SB, EG/R) in Bezug auf erzeugtes Lungenvolumen und auftretende Magenblähung zu vergleichen. Dabei berücksichtigten wir die Veränderung der Lungencompliance und des UÖSD. In den ersten Minuten nach einem Herzkreislaufstillstand nehmen sowohl die Compliance der Lunge als auch UÖSD ab.

Zwei Hypothesen galt es zu testen:

\section{Hypothese 1}

Zwischen den drei Testapparaturen gibt es keine Unterschiede in der Grösse der Atemzugvolumen.

\section{Hypothese 2}

Bei Gebrauch des Easy Grip mit Reservoir ist die Beatmungsleistung gleichwertig oder vergleichsweise gut wie bei Gebrauch des SMART BAG®. Wir erwarten eine vergleichbare Magenblähung und ein ausreichendes Tidalvolumen selbst im Schlimmstfall-Szenario bei einer Lungencompliance von $500 \mathrm{ml} / \mathrm{kPa}$ und einem UÖSD von $0.5 \mathrm{kPa}$. 


\section{Material und Methode}

\subsection{Probanden}

Für die Versuchsdurchführungen wurden 44 Zahnärzte (Zentrum für Zahnmedizin, Universität Zürich) des Abschlussjahrganges 2006 angefragt, ob sie an der Studie teilnehmen möchten. 7 Frauen und 7 Männer willigten ein. Die Versuchsdurchführungen begannen im Dezember 2006 und dauerten bis März 2007. Alle Teilnehmer hatten soeben das Staatsexamen absolviert. Unter innen gab es drei Probanden, welche vor dem Zahnmedizinstudium bereits ein Humanmedizinstudium abgeschlossen und auch schon als Assistenzärzte gearbeitet hatten.

Während dem Zahnmedizinstudium war jeder einzelne Proband mindestens einmal in CPR (kardiopulmonale Reanimation) an Puppen ausgebildet worden. Diese Ausbildung lag weniger als 1 Jahr und mehr als 6 Monate vor diesen Versuchen zurück. Keine der Testpersonen war bisher schon einmal mit einer CPR Situation im Alltag konfrontiert worden.

Die Studie wurde durch die kantonale Ethikkommission von Zürich genehmigt. Mittels Unterschrift gaben die Teilnehmer ihr Einverständnis für die anonymisierte Verwendung der Videoaufnahmen der Wiederbelebungsmassnahmen in wissenschaftlichen Erhebungen und Publikationen.

Alle Versuche wurden auf der Poliklinik für Orale Chirurgie am ZZM (Universität Zürich) durchgeführt.

\subsection{Testsysteme}

Es wurden drei verschiedene Beatmungssysteme verwendet und in einem randomisiertem CrossoverDesign miteinander verglichen: ein Easy Grip® Einwegatembeutel (EG), ein SMART BAG® $(S B)$ und ein Easy Grip® Einwegatembeutel mit vorgeschaltetem Reservoir (EG/R). 


\section{Easy Grip ${ }^{\circledR}(E G)$}

Als erste Vorrichtung wurde ein Easy Grip ${ }^{\circledR}$ (O-Two Medical Technologies Inc, Mississauga, Ontario, Canada) Einwegatembeutel verwendet. Dieses System diente als Standardvorrichtung, da es aktuell in der zahnärztlichen Praxis am häufigsten gebraucht wird (Abbildung 1).

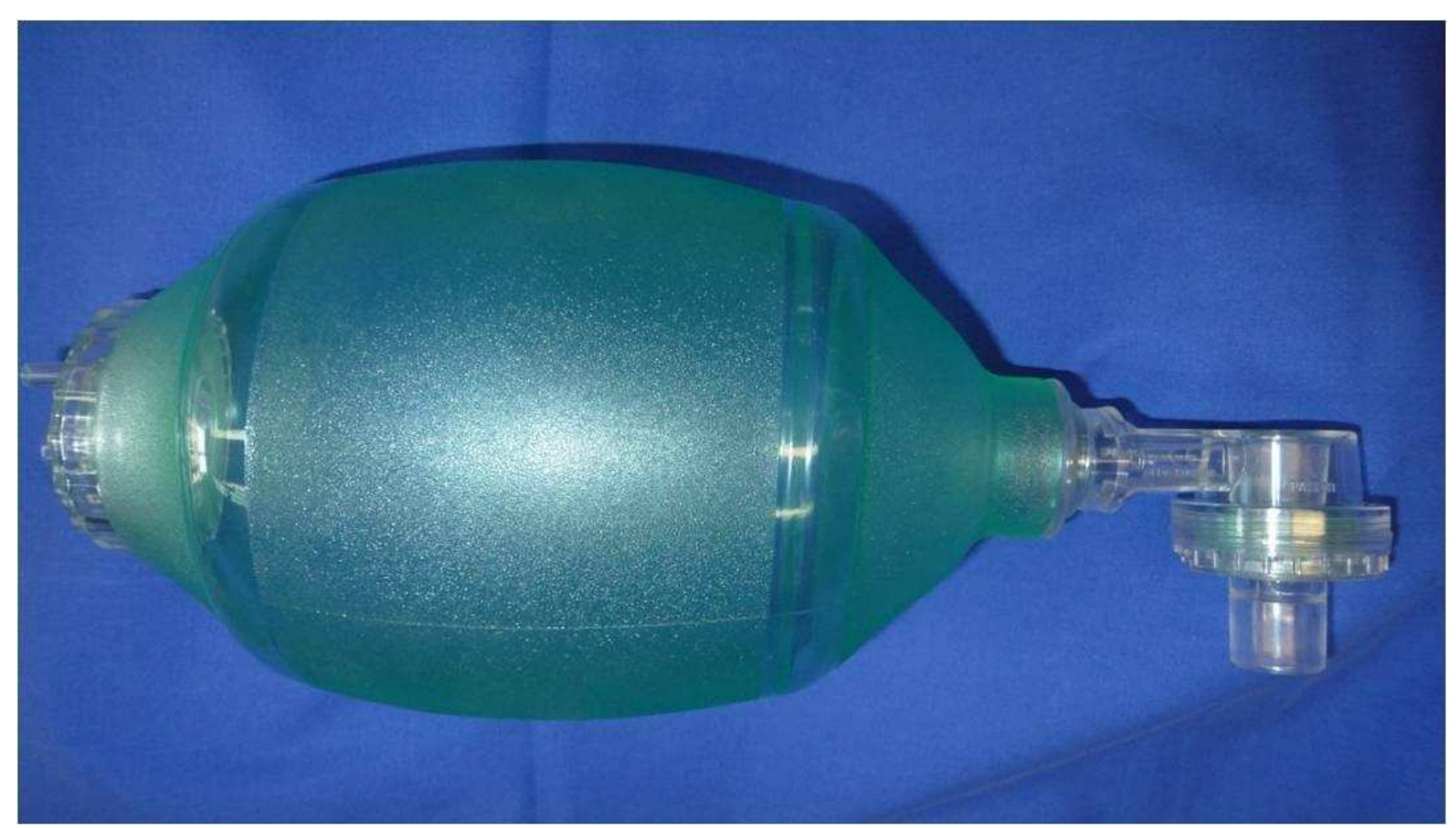

Abbildung 1: Easy Grip® Einwegbeatmungsbeutel

\section{Technische Daten:}

- Atembeutelvolumen $1700 \mathrm{ml}$

- inspiratorischer Widerstand $3.3 \mathrm{~cm} \mathrm{H} 2 \mathrm{O}(0.33 \mathrm{kPa})$

- exspiratorischer Widerstand $2.2 \mathrm{~cm} \mathrm{H} 2 \mathrm{O}(0.22 \mathrm{kPa})$

- Totraumvolumen $7 \mathrm{ml}$

(Alle technischen Angaben mit Toleranz von $+/-10 \%$ ) 


\section{SMART BAG® (SB)}

Als zweite Vorrichtung wurde der SMART BAG® (O-Two Medical Technologies Inc, Mississauga, Ontario, Canada), ein spitzenfluss- und druckbegrenzender Einwegatembeutel verwendet (Abbildung 2). Ein spezielles druckgegensteuerndes Ventil limitiert den inspiratorischen Gasfluss und somit den Atemwegsspitzendruck (A. von Goedecke, P. Paal 2006), was das Risiko einer Magenblähung herabsetzt. Zwei weitere Eigenschaften tragen ausserdem dazu bei, die beatmende Person auf einen zu hohen Gasfluss aufmerksam zu machen. Bei zu starker Kompression des Beutels erscheint am Hals des SB ein roter Indikator als Warnung. Ausserdem nimmt der Widerstand des Beutels bei zu grossem Druck zu und er kann bei gleichbleibender Beatmung nicht mehr im gleichen Masse komprimiert werden.

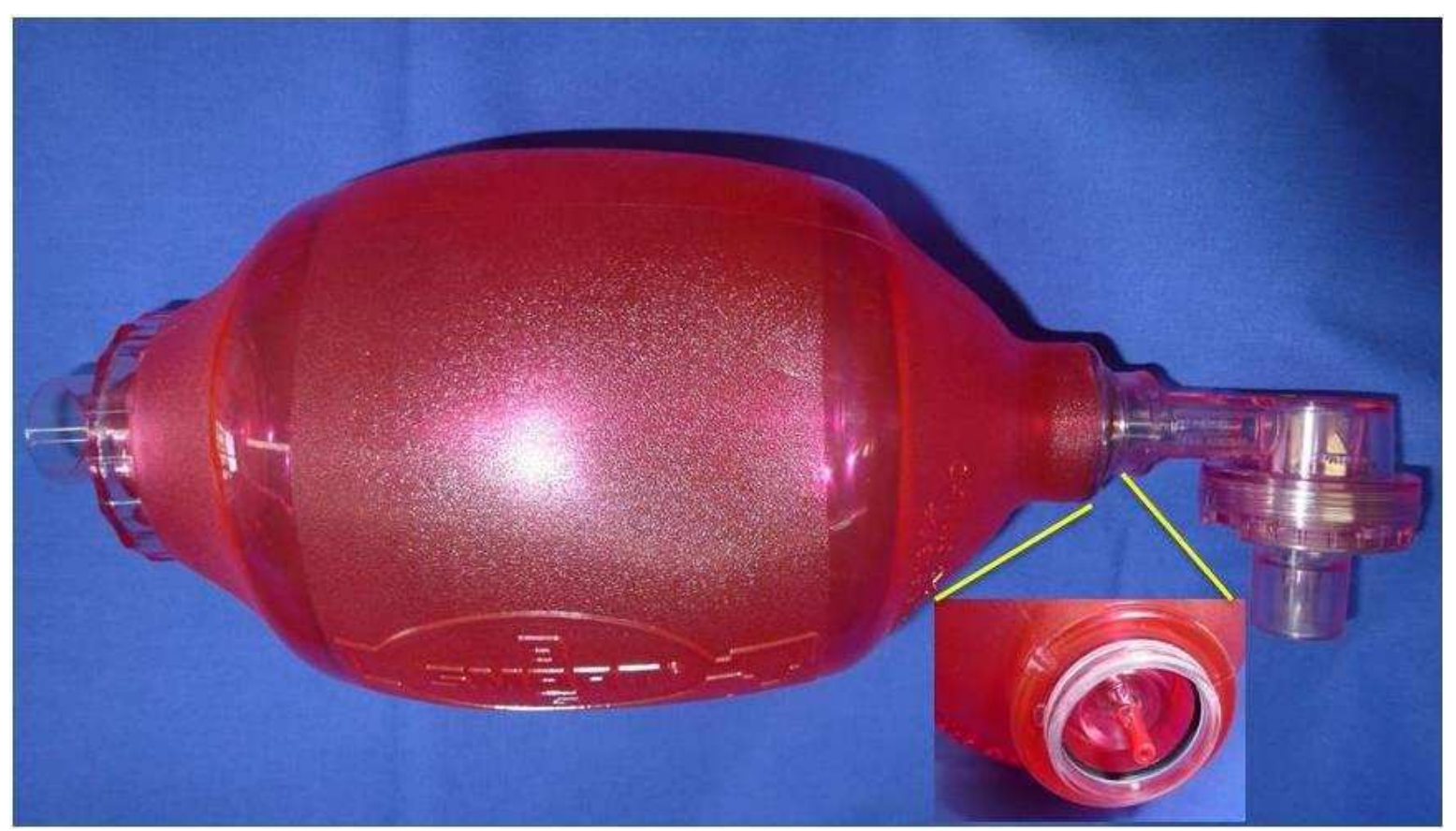

Abbildung 2: SMART BAG®

\section{Technische Daten:}

- Atembeutelvolumen $1700 \mathrm{ml}$

- inspiratorischer Widerstand $3.3 \mathrm{~cm} \mathrm{H} 2 \mathrm{O}(0.33 \mathrm{kPa})$

- exspiratorischer Widerstand $2.2 \mathrm{~cm} \mathrm{H} 2 \mathrm{O}(0.22 \mathrm{kPa})$

- Totraumvolumen $7 \mathrm{ml}$

(Alle technischen Angaben mit Toleranz von $+/-10 \%$ ) 


\section{Easy Grip ${ }^{\circledR}$ mit Reservoir (EG/R)}

Als dritte Vorrichtung wurde wiederum ein Einwegatembeutel der Marke Easy Grip® (O-Two Medical Technologies Inc, Mississauga, Ontario, Canada) verwendet. Zusätzlich konnte jedoch mit Hilfe eines T-Stücks ein 0.5l Rüsch Atembeutel (Willy RÜSCH GmbH, Kernen i.R., Deutschland, Abbildung 3) vorgeschaltet und somit ein Windkessel erzeugt werden (Abbildung 4). Bei zu schneller und/oder zu starker Ventilation nimmt dieses Reservoir zusätzlich Luft auf, um einem exzessivem Beatmungsdruck vorzubeugen.

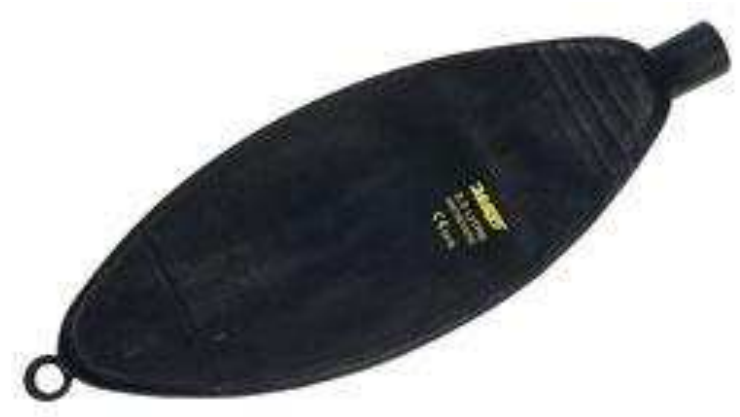

Abbildung 3: 0.5I Rüsch Atembeutel

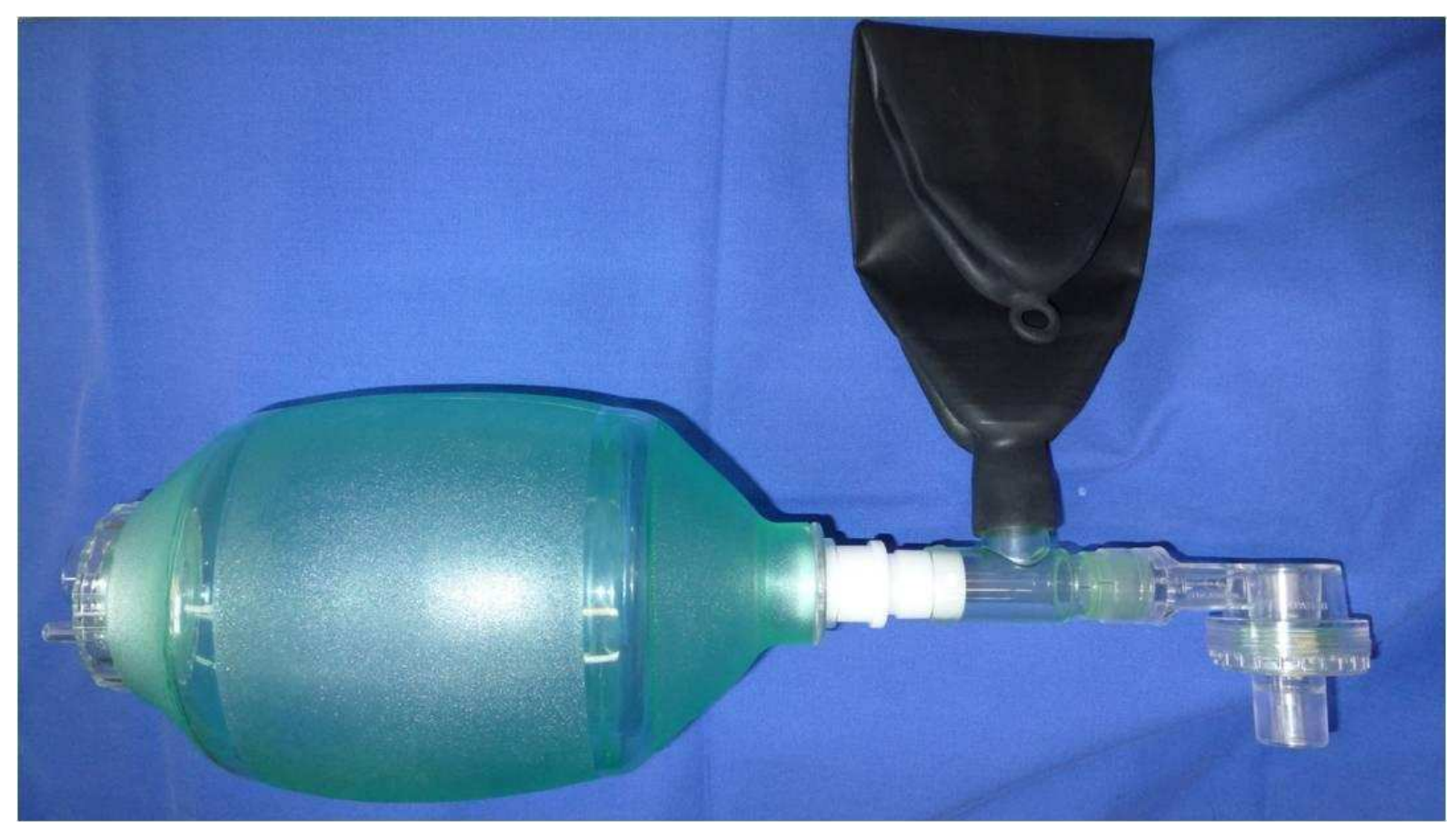

Abbildung 4: Easy Grip® mit vorgeschaltetem Atembeutel 


\subsection{Versuchsaufbau}

Für die Versuchsdurchführung verwendeten wir einen Mini Ventilation Training Analyzer, welcher über vier Anschlüsse verfügt. An einem Anschluss wurde eine Testlunge befestigt, ein anderer Anschluss wurde zur Simulation des unteren Ösophagussphinkterdrucks benutzt und an den dritten Anschluss wurden die drei Beatmungsbeutel adaptiert. Der vierte Anschluss wurde für unsere Versuchszwecke nicht benötigt. Der Widerstand der künstlichen Atemwege und die Compliance der Lunge wurden vorgängig mit Hilfe eines Evita XL Beatmungsgerätes (Draeger Luebeck, Germany) bestimmt. Dieses Gerät kann Widerstände bis $600 \mathrm{mbar} / \mathrm{l} / \mathrm{s}$ und eine Compliance bis $3000 \mathrm{ml} / \mathrm{kPa}$ messen.

Die Compliance unserer Testlunge (O-TWO Systems) betrug $700 \mathrm{ml} / \mathrm{kPa}$ bei einem Volumen von 800ml. Mit Hilfe eines Gummibandes, welches immer an der gleichen Stelle positioniert wurde, konnte die Compliance auf $500 \mathrm{ml} / \mathrm{kPa}$ bei einem Volumen von $900 \mathrm{ml}$ reduziert werden (Abbildung 5). Der Widerstand der Atemwege betrug $10 \mathrm{mbar} / \mathrm{l} / \mathrm{s}(1 \mathrm{kPa} / \mathrm{l} / \mathrm{s})$.

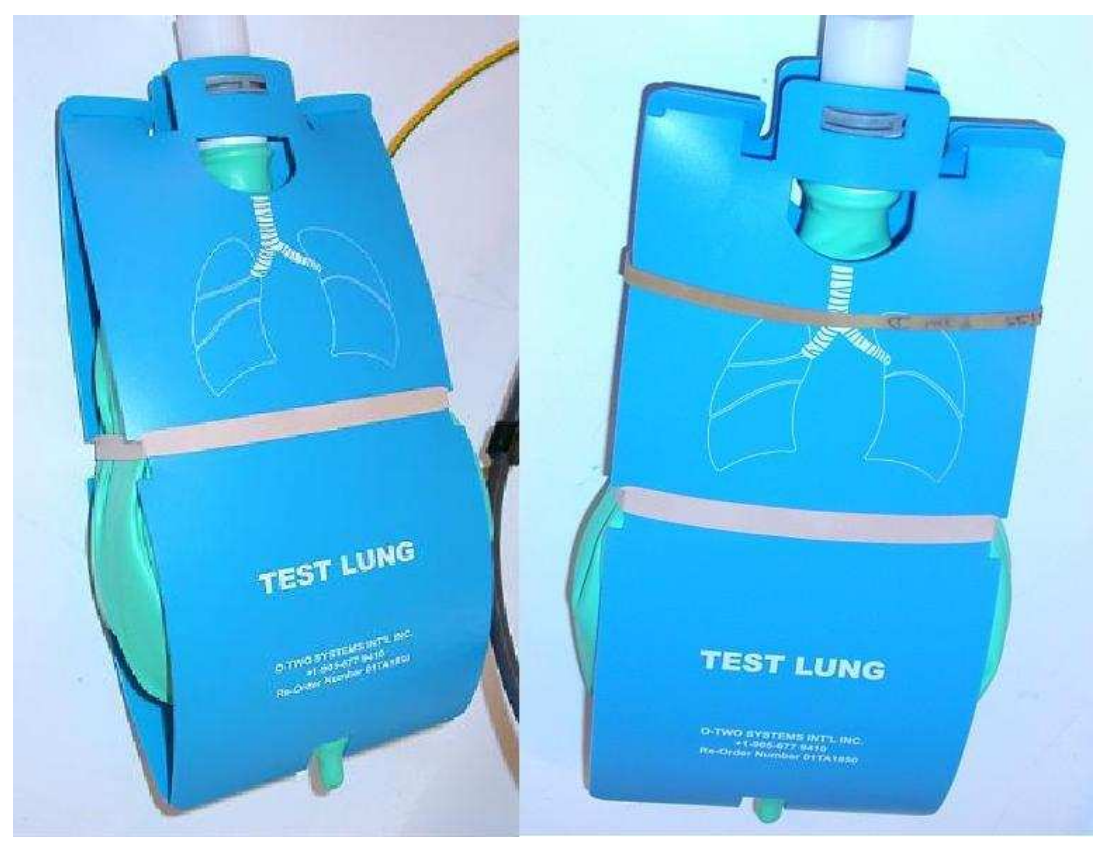

Abbildung 5: Testlunge auf der linken Seite mit hoher Compliance (700ml/kPa bei $800 \mathrm{ml}$ Volumen), Testlunge auf der rechten Seite mit niedriger Compliance $(500 \mathrm{ml} / \mathrm{kPa}$ bei $900 \mathrm{ml}$ Volumen).

Der Magen wurde durch zwei Wasserflaschen imitiert und mit dem Mini Ventilation Training Analyzer verbunden (Abbildung 6). In der einen Flasche befanden sich $5 \mathrm{~cm} \mathrm{H}_{2} \mathrm{O}$ und in der anderen Flasche $15 \mathrm{~cm} \mathrm{H}_{2} \mathrm{O}$. So konnte ein UÖSD von $0.5 \mathrm{kPa}$ bzw. $1.5 \mathrm{kPa}$ simuliert werden. 
Mit Hilfe von vorgeschalteten Volumeter konnten einerseits die Tidalvolumina und andererseits die Magenblähung gemessen werden (Abbildungen 7 und 8).

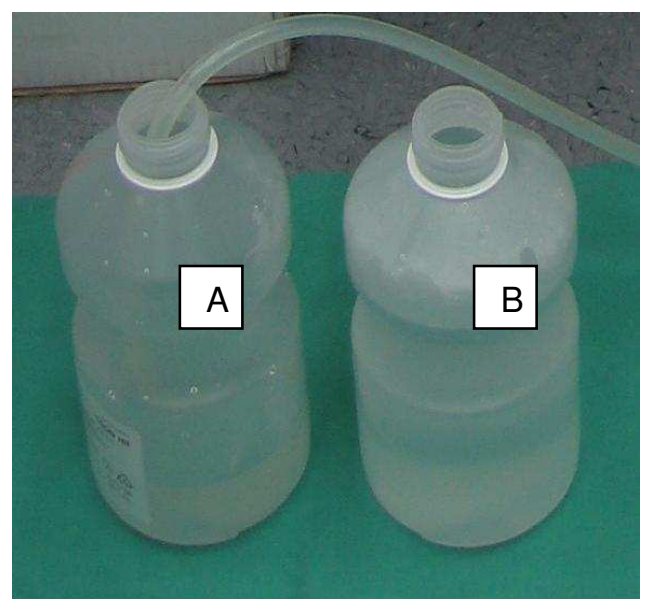

Abbildung 6: Simulation von UÖSD; Flasche A mit $5 \mathrm{~cm} \mathrm{H}_{2} \mathrm{O}(0.5 \mathrm{kPa})$, Flasche B mit $15 \mathrm{~cm} \mathrm{H}_{2} \mathrm{O}$ $(1.5 \mathrm{kPa})$

Anschluss für die drei

Testvorrichtungen
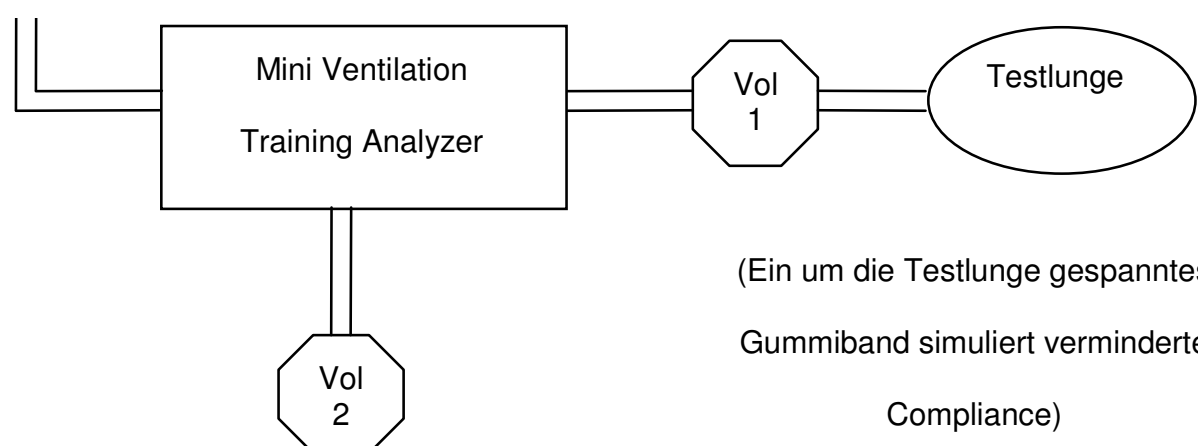

(Ein um die Testlunge gespanntes

Gummiband simuliert verminderte

Compliance)

Wasserspiegel

simuliert UÖSD

Abbildung 7: Schema des Versuchsaufbaus; Volumeter 1 (Vol1) misst die Tidalvolumina, Volumeter 2 (Vol2) die Magenblähung; ein Wasserspiegel simuliert UÖSD; die Compliance der Testlunge wird durch ein Gummiband vermindert. 


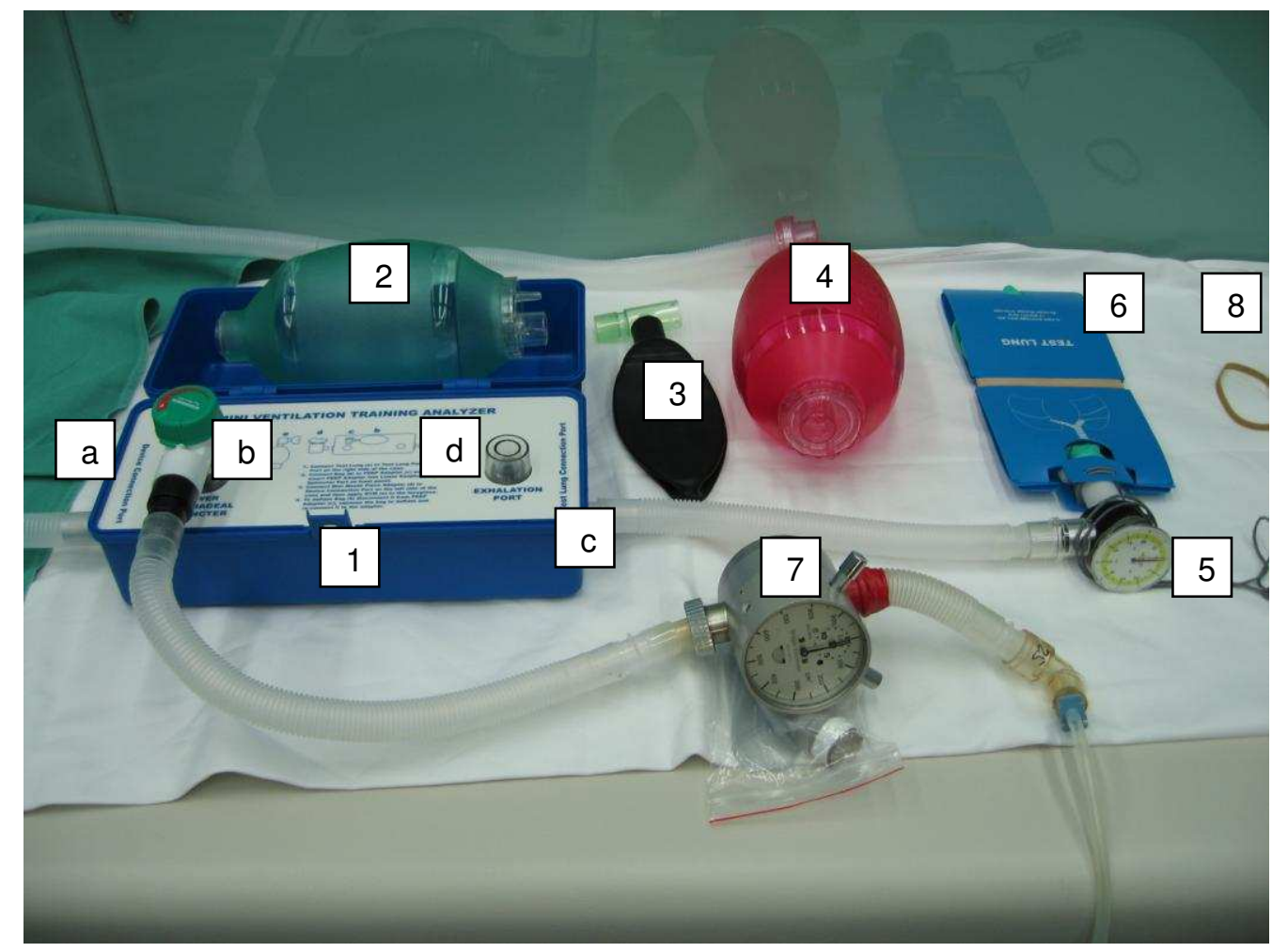

Abbildung 8: Versuchsaufbau

1: Mini Ventilation Training Analyzer

a: Anschluss für die Beatmungsbeutel

b: Anschluss für die Wasserflasche, welche den UÖSD simuliert (siehe unten)

c: Anschluss für die Testlunge

d: vierter Anschluss (für diese Versuchsreihe nicht benötigt)

2: Easy Grip ${ }^{\circledR}$ Beatmungsbeutel (EG)

3: $\quad 0.5$ I Rüsch Atembeutel (Reservoir)

4: $\quad$ SMART BAG® $(\mathrm{SB})$

5: Volumeter 1 (misst Menge an Luft, welche in die Lunge gelangt)

6: Testlunge von O-TWO Systems

7: Volumeter 1 (misst Menge an Luft, welche in den Magen gelangt)

8: 2. Gummiband (wird um Lunge gelegt, um Compliance zu reduzieren) 


\subsection{Methode}

Die Probanden wurden vorgängig weder über das Risiko der Magenblähung, noch über die Notwendigkeit der suffizienten Lungeninflation informiert. Sie mussten die Beatmung einzig nach ihrer eigenen Einschätzung durchführen und wurden nur über die Bewegungen der Testlunge aufmerksam gemacht. Dabei wussten sie aber nicht, welche Inflationsstärke eine genügende Beatmung simuliert. Sie durften mit einer oder beiden Händen beatmen, konnten während der Beatmung die Bewegung der Testlunge verfolgen und hörten das Einströmen von Luft in Wasser (Simulation von UÖSD), ohne aber über dessen Bedeutung in Kenntnis gesetzt worden zu sein. Nachfolgende Teilnehmer der Studie durften nicht bei den vorhergehenden Versuchen zusehen, damit eine eventuelle Beeinflussung ausgeschlossen werden konnte.

Für jeden der 12 Versuchsaufbauten mussten jeweils 10 Beatmungen vollzogen werden. Zwischen den Versuchen wurden einzelne Parameter verändert: Austausch des Beatmungsbeutels (EG, SB und $\mathrm{EG} / \mathrm{R})$, Veränderung von UÖSD $(0.5 \mathrm{kPa}$ oder $1.5 \mathrm{kPa})$ und Erhöhung oder Verminderung der Lungencompliance $(700 \mathrm{ml} / \mathrm{kPa}$ oder $500 \mathrm{ml} / \mathrm{kPa})$. Die Abfolge der einzelnen Versuchsaufbauten erfolgte in einem randomisierten Crossover-Design.

\begin{tabular}{|c|c|c|c|c|}
\cline { 2 - 5 } \multicolumn{1}{c|}{} & \multicolumn{2}{c|}{ Compliance hoch $(700 \mathrm{ml} / \mathrm{kPa})$} & \multicolumn{2}{c|}{ Compliance niedrig $(500 \mathrm{ml} / \mathrm{kPa})$} \\
\hline UÖSD & $0.5 \mathrm{kPa}$ & $1.5 \mathrm{kPa}$ & $0.5 \mathrm{kPa}$ & $1.5 \mathrm{kPa}$ \\
\hline Easy Grip (EG) & $A$ & $B$ & $G$ & $\mathrm{H}$ \\
\hline SMART BAG (SB) & $C$ & $D$ & $I$ & $K$ \\
\hline EG mit Reservoir (EG/R) & $E$ & $F$ & $L$ & $M$ \\
\hline
\end{tabular}

Tabelle 1: Einzelne Versuchsdurchgänge (A-M) in der Übersicht

Die einzelnen Versuchsdurchführungen wurden mit Hilfe einer Videokamera aufgenommen und später ausgewertet. Die Buchstaben A - M wurden vor jedem Durchgang aufgezeichnet und dienten dazu, die einzelnen Parameter den jeweiligen Versuchsaufbauten zu zu teilen. 
4. Resultate

\subsection{Tabellarische Darstellung der Testergebnisse}

4.1.1. Lungenvolumina bei hoher Lungencompliance (Tabellen 2 und 3)

\begin{tabular}{|c|c|c|c|}
\hline \multicolumn{4}{|c|}{ Lungencompliance hoch (700ml/kPa), UÖSD 0.5 kPa } \\
\hline Proband & $E G$ & $S B$ & $E G / R$ \\
\hline 1 & 475 & 437.5 & 237.5 \\
\hline 2 & 612.5 & 600 & 500 \\
\hline 3 & 675 & 550 & 487.5 \\
\hline 4 & 900 & 425 & 600 \\
\hline 5 & 900 & 837.5 & 425 \\
\hline 6 & 1000 & 612.5 & 650 \\
\hline 7 & 650 & 325 & 312.5 \\
\hline 8 & 600 & 487.5 & 287.5 \\
\hline 9 & 662.5 & 337.5 & 400 \\
\hline 10 & 662.5 & 412.5 & 375 \\
\hline 11 & 662.5 & 500 & 500 \\
\hline 12 & 800 & 550 & 450 \\
\hline 13 & 650 & 412.5 & 425 \\
\hline 14 & 462.5 & 300 & 250 \\
\hline Median & 662.5 & 462.5 & 425 \\
\hline \multicolumn{4}{|c}{} \\
\hline
\end{tabular}

Tabelle 2: Medianwerte der Lungenvolumina (in $\mathrm{ml}$ ) aller Probanden bei hoher Lungencompliance $(700 \mathrm{ml} / \mathrm{kPa})$ und tiefem UÖSD $(0.5 \mathrm{kPa})$

\begin{tabular}{|c|c|c|c|}
\hline \multicolumn{4}{|c|}{ Lungencompliance hoch (700ml/kPa). UÖSD 1.5kPa } \\
\hline Proband & $E G$ & $S B$ & $E G / R$ \\
\hline 1 & 450 & 437.5 & 300 \\
\hline 2 & 700 & 675 & 750 \\
\hline 3 & 737.5 & 437.5 & 475 \\
\hline 4 & 975 & 400 & 600 \\
\hline 5 & 975 & 950 & 450 \\
\hline 6 & 950 & 725 & 750 \\
\hline 7 & 650 & 450 & 400 \\
\hline 8 & 662.5 & 575 & 350 \\
\hline 9 & 687.5 & 450 & 412.5 \\
\hline 10 & 700 & 425 & 400 \\
\hline 11 & 700 & 525 & 575 \\
\hline 12 & 800 & 650 & 550 \\
\hline 13 & 750 & 462.5 & 450 \\
\hline 14 & 625 & 487.5 & 350 \\
\hline Median & 700 & 475 & 450 \\
\hline \multicolumn{4}{|c}{} \\
\hline
\end{tabular}

Tabelle 3: Medianwerte der Lungenvolumina (in $\mathrm{ml}$ ) aller Probanden bei hoher Lungencompliance $(700 \mathrm{ml} / \mathrm{kPa})$ und hohem UÖSD $(1.5 \mathrm{kPa})$ 
4.1.2. Lungenvolumina bei tiefer Lungencompliance (Tabellen 4 und 5)

\begin{tabular}{|c|c|c|c|}
\hline \multicolumn{4}{|c|}{ Lungencompliance niedrig $(500 \mathrm{ml} / \mathrm{kPa})$, UÖSD 0.5kPa } \\
\hline Proband & $E G$ & $S B$ & $E G / R$ \\
\hline 1 & 437.5 & 400 & 300 \\
\hline 2 & 900 & 587.5 & 587.5 \\
\hline 3 & 675 & 387.5 & 450 \\
\hline 4 & 875 & 375 & 550 \\
\hline 5 & 925 & 650 & 500 \\
\hline 6 & 1000 & 637.5 & 575 \\
\hline 7 & 375 & 362.5 & 200 \\
\hline 8 & 537.5 & 500 & 300 \\
\hline 9 & 675 & 300 & 337.5 \\
\hline 10 & 725 & 450 & 425 \\
\hline 11 & 750 & 525 & 450 \\
\hline 12 & 750 & 550 & 412.5 \\
\hline 13 & 750 & 475 & 450 \\
\hline 14 & 450 & 200 & 200 \\
\hline Median & 737.5 & 462.5 & 437.5 \\
\hline
\end{tabular}

Tabelle 4: Medianwerte der Lungenvolumina (in $\mathrm{ml}$ ) aller Probanden bei tiefer Lungencompliance $(500 \mathrm{ml} / \mathrm{kPa})$ und tiefem UÖSD $(0.5 \mathrm{kPa})$

\begin{tabular}{|c|c|c|c|}
\hline \multicolumn{4}{|c|}{ Lungencompliance niedrig $(500 \mathrm{ml} / \mathrm{kPa}), U O ̈ S D 1.5 \mathrm{kPa}$} \\
\hline Proband & $E G$ & $S B$ & $E G / R$ \\
\hline 1 & 437.5 & 400 & 312.5 \\
\hline 2 & 700 & 650 & 612.5 \\
\hline 3 & 725 & 450 & 450 \\
\hline 4 & 950 & 400 & 600 \\
\hline 5 & 937.5 & 850 & 500 \\
\hline 6 & 1100 & 737.5 & 750 \\
\hline 7 & 500 & 500 & 300 \\
\hline 8 & 650 & 650 & 325 \\
\hline 9 & 700 & 425 & 412.5 \\
\hline 10 & 700 & 500 & 400 \\
\hline 11 & 750 & 650 & 537.5 \\
\hline 12 & 762.5 & 700 & 512.5 \\
\hline 13 & 750 & 537.5 & 475 \\
\hline 14 & 525 & 375 & 262.5 \\
\hline Median & $\mathbf{7 1 2 . 5}$ & $\mathbf{5 1 8 . 7 5}$ & $\mathbf{4 6 2 . 5}$ \\
\hline \multicolumn{4}{|r}{} \\
\hline
\end{tabular}

Tabelle 5: Medianwerte der Lungenvolumina (in $\mathrm{ml}$ ) aller Probanden bei tiefer Lungencompliance $(500 \mathrm{ml} / \mathrm{kPa})$ und hohem UÖSD $(1.5 \mathrm{kPa})$ 
4.1.3. Magenvolumina bei hoher Lungencompliance (Tabellen 6 und 7)

\begin{tabular}{|c|c|c|c|}
\hline \multicolumn{4}{|c|}{ Lungencompliance hoch (700ml/kPa), UÖSD 0.5kPa } \\
\hline Proband & $E G$ & $S B$ & $E G / R$ \\
\hline 1 & 50 & 50 & 0 \\
\hline 2 & 100 & 100 & 62.5 \\
\hline 3 & 112.5 & 75 & 75 \\
\hline 4 & 175 & 50 & 100 \\
\hline 5 & 175 & 175 & 50 \\
\hline 6 & 225 & 150 & 100 \\
\hline 7 & 100 & 0 & 12.5 \\
\hline 8 & 75 & 50 & 0 \\
\hline 9 & 100 & 25 & 50 \\
\hline 10 & 100 & 50 & 50 \\
\hline 11 & 125 & 100 & 100 \\
\hline 12 & 150 & 112.5 & 75 \\
\hline 13 & 137.5 & 62.5 & 50 \\
\hline 14 & 375 & 300 & 175 \\
\hline Median & $\mathbf{1 1 8 . 7 5}$ & $\mathbf{6 8 . 7 5}$ & $\mathbf{5 6 . 2 5}$ \\
\hline
\end{tabular}

Tabelle 6: Medianwerte der Magenvolumina (in $\mathrm{ml}$ ) aller Probanden bei hoher Lungencompliance $(700 \mathrm{ml} / \mathrm{kPa})$ und tiefem UÖSD $(0.5 \mathrm{kPa})$

\begin{tabular}{|c|c|c|c|}
\hline \multicolumn{5}{|c|}{ Lungencompliance hoch (700ml/kPa), UÖSD 1.5kPa } \\
\hline Proband & $E G$ & $S B$ & $E G / R$ \\
\hline 1 & 0 & 0 & 0 \\
\hline 2 & 0 & 0 & 25 \\
\hline 3 & 50 & 0 & 0 \\
\hline 4 & 137.5 & 0 & 25 \\
\hline 5 & 50 & 25 & 0 \\
\hline 6 & 100 & 0 & 37.5 \\
\hline 7 & 0 & 0 & 0 \\
\hline 8 & 0 & 0 & 0 \\
\hline 9 & 62.5 & 0 & 0 \\
\hline 10 & 50 & 0 & 0 \\
\hline 11 & 25 & 0 & 0 \\
\hline 12 & 75 & 0 & 25 \\
\hline 13 & 50 & 0 & 0 \\
\hline 14 & 175 & 25 & 25 \\
\hline Median & $\mathbf{5 0}$ & $\mathbf{0}$ & $\mathbf{0}$ \\
\hline
\end{tabular}

Tabelle 7: Medianwerte der Magenvolumina (in $\mathrm{ml}$ ) aller Probanden bei hoher Lungencompliance $(700 \mathrm{ml} / \mathrm{kPa})$ und hohem UÖSD (1.5kPa) 
4.1.4. Magenvolumina bei tiefer Lungencompliance (Tabellen 8 und 9)

\begin{tabular}{|c|c|c|c|}
\hline \multicolumn{4}{|c|}{ Lungencompliance niedrig $(500 \mathrm{ml} / \mathrm{kPa})$, ÜSD 0.5kPa } \\
\hline Proband & $E G$ & $S B$ & $E G / R$ \\
\hline 1 & 50 & 50 & 0 \\
\hline 2 & 212.5 & 125 & 125 \\
\hline 3 & 125 & 75 & 75 \\
\hline 4 & 200 & 50 & 100 \\
\hline 5 & 200 & 200 & 100 \\
\hline 6 & 200 & 200 & 100 \\
\hline 7 & 25 & 25 & 0 \\
\hline 8 & 100 & 75 & 25 \\
\hline 9 & 100 & 25 & 25 \\
\hline 10 & 125 & 100 & 50 \\
\hline 11 & 150 & 0 & 75 \\
\hline 12 & 0 & 125 & 50 \\
\hline 13 & 150 & 100 & 75 \\
\hline 14 & 450 & 375 & 200 \\
\hline Median & $\mathbf{1 3 7 . 5}$ & $\mathbf{8 7 . 5}$ & $\mathbf{7 5}$ \\
\hline
\end{tabular}

Tabelle 8: Medianwerte der Magenvolumina (in $\mathrm{ml}$ ) aller Probanden bei tiefer Lungencompliance $(500 \mathrm{ml} / \mathrm{kPa})$ und tiefem UÖSD $(0.5 \mathrm{kPa})$

\begin{tabular}{|c|c|c|c|}
\hline \multicolumn{4}{|c|}{ Lungencompliance niedrig $(500 \mathrm{ml} / \mathrm{kPa}), U$ ÖSD $1.5 \mathrm{kPa}$} \\
\hline Proband & $E G$ & $S B$ & $E G / R$ \\
\hline 1 & 0 & 0 & 0 \\
\hline 2 & 25 & 0 & 0 \\
\hline 3 & 75 & 0 & 0 \\
\hline 4 & 150 & 0 & 50 \\
\hline 5 & 50 & 0 & 0 \\
\hline 6 & 150 & 0 & 25 \\
\hline 7 & 0 & 0 & 0 \\
\hline 8 & 0 & 0 & 0 \\
\hline 9 & 75 & 0 & 0 \\
\hline 10 & 50 & 0 & 0 \\
\hline 11 & 37.5 & 0 & 0 \\
\hline 12 & 50 & 0 & 0 \\
\hline 13 & 75 & 0 & 0 \\
\hline 14 & 200 & 100 & 25 \\
\hline Median & $\mathbf{5 0}$ & $\mathbf{0}$ & $\mathbf{0}$ \\
\hline
\end{tabular}

Tabelle 9: Medianwerte der Magenvolumina (in $\mathrm{ml}$ ) aller Probanden bei tiefer Lungencompliance $(500 \mathrm{ml} / \mathrm{kPa})$ und hohem UÖSD (1.5kPa) 


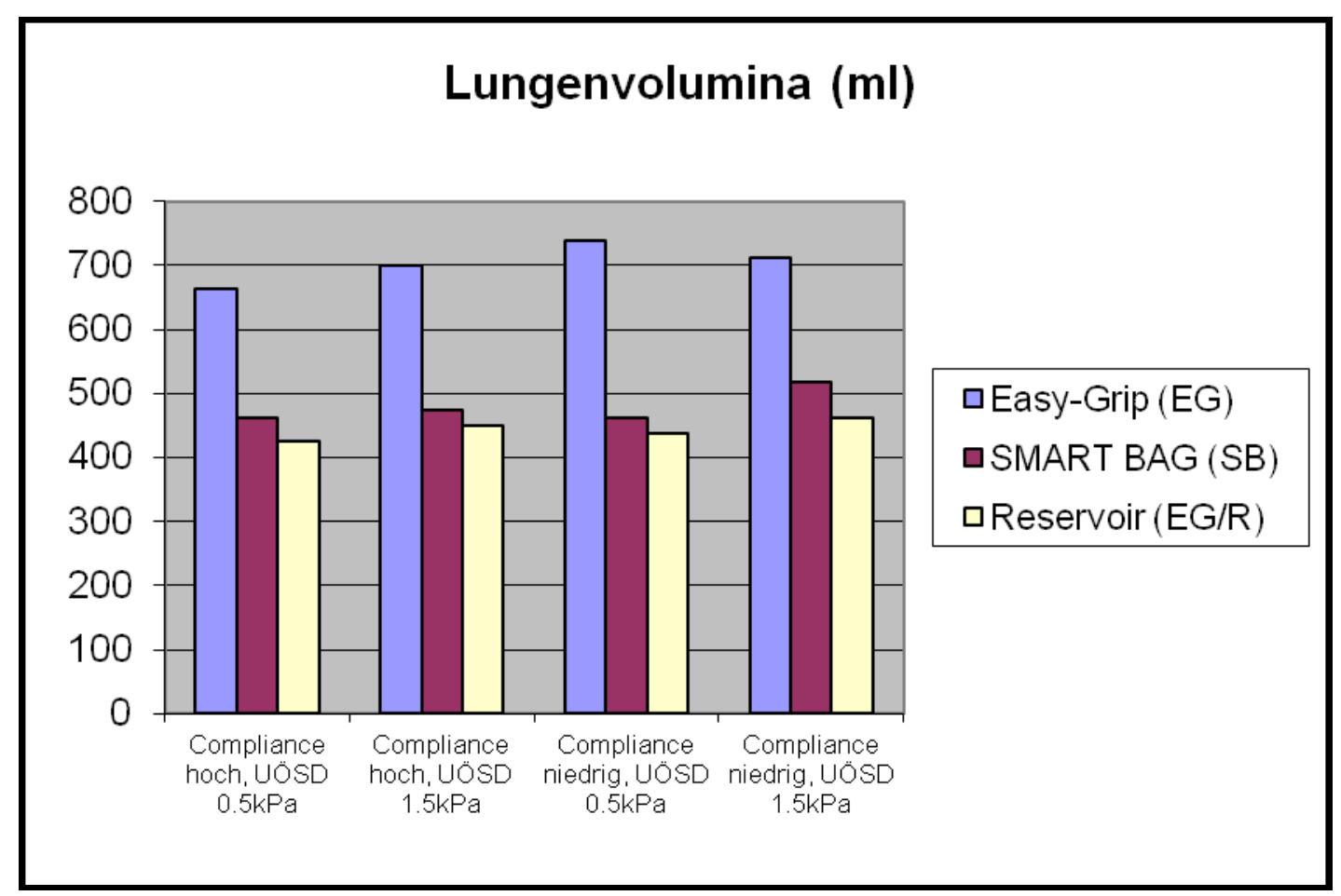

Grafik 1: Medianwerte der Lungenvolumina (in $\mathrm{ml}$ ) in Abhängigkeit von Lungencompliance und UÖSD

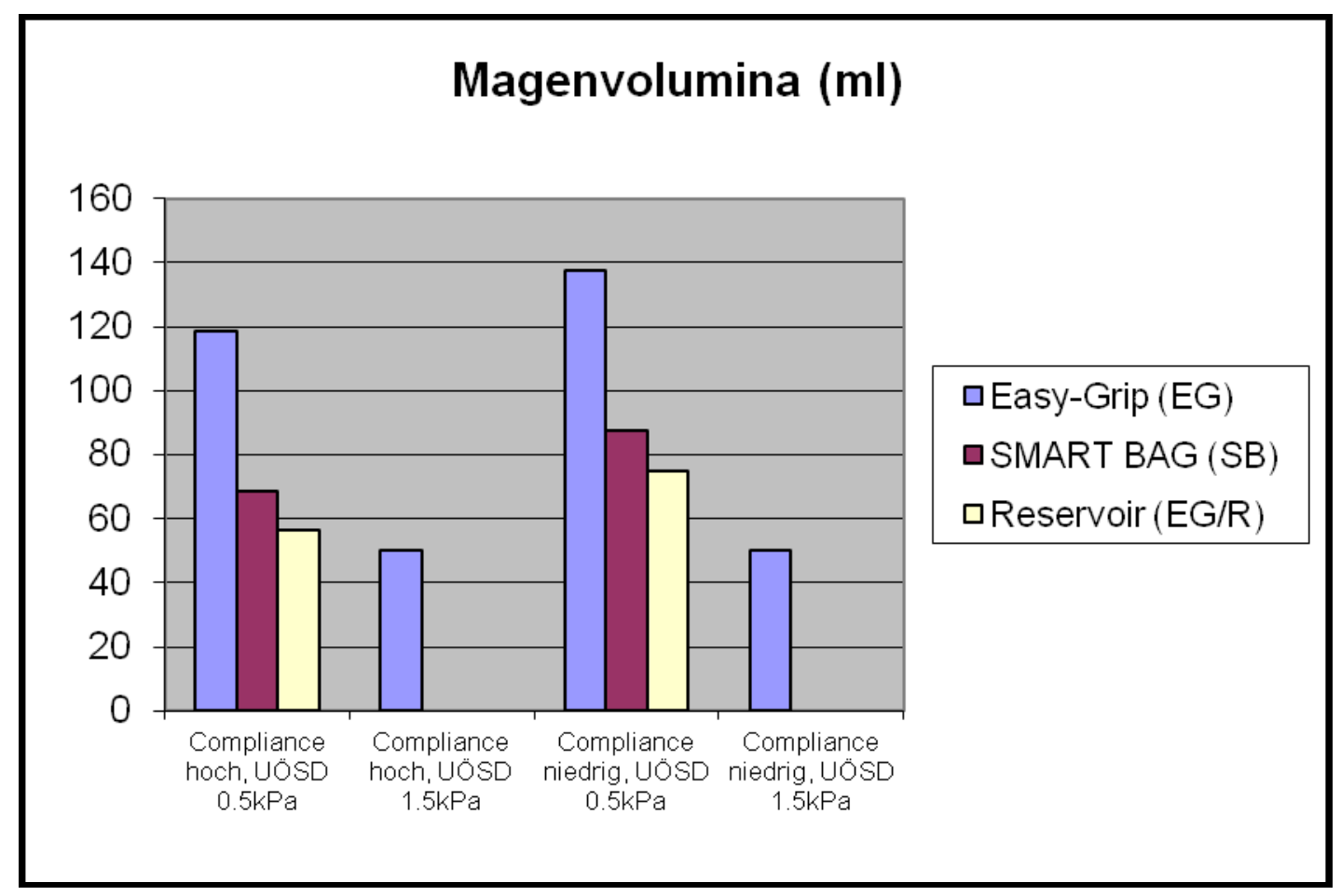

Grafik 2: Medianwerte der Magenvolumina (in $\mathrm{ml}$ ) in Abhängigkeit von Lungencompliance und UÖSD 


\subsection{Statistik}

Für jede Versuchsanordnung und jeden Probanden wurden die Median- und Quartilwerte der Lungenbzw. Magenvolumina ausgerechnet.

Um die Werte miteinander vergleichen zu können, wendeten wir den Vorzeichen-Test von Wilcoxon an. Dieser Test ist ein nichtparametrischer Test in der Statistik, bei welchem gepaarte Stichproben auf eine Veränderung oder eine Tendenz auf Signifikanz überprüft werden.

Nach Bonferroni-Korrektur für multiple Vergleiche wurde ein Signifikanzniveau von $p=0.016$ errechnet.

\subsubsection{Lungenvolumina (in $\mathrm{ml}$ )}

\begin{tabular}{|c|c|c|c|}
\hline \multicolumn{4}{|c|}{$\begin{array}{l}\text { Lungencompliance hoch, } \\
\text { UÖSD }=0.5 \mathrm{kPa}\end{array}$} \\
\hline & $E G$ & SB & $E G / R$ \\
\hline Max & 1000 & 837.5 & 650 \\
\hline $75 \% Q$ & 769 & 550 & 497 \\
\hline Median & 662.5 & 462.5 & 425 \\
\hline $25 \% Q$ & 622 & 412.5 & 328 \\
\hline Min & 462.5 & 300 & 237.5 \\
\hline$p$ [EG vs SB] & \multicolumn{2}{|c|}{0.0001} & \\
\hline$p[E G$ vs $E G / R]$ & \multicolumn{3}{|c|}{0.0001} \\
\hline $\mathrm{p}[\mathrm{SB}$ vs $\mathrm{EG} / \mathrm{R}]$ & & \multicolumn{2}{|c|}{0.1099} \\
\hline
\end{tabular}

\begin{tabular}{|c|c|c|c|}
\hline \multicolumn{4}{|c|}{$\begin{array}{l}\text { Lungencompliance hoch, } \\
\text { UÖSD }=1.5 \mathrm{kPa}\end{array}$} \\
\hline & $E G$ & SB & $E G / R$ \\
\hline Max & 975 & 950 & 750 \\
\hline $75 \% Q$ & 787.5 & 631.5 & 569 \\
\hline Median & 700 & 462.5 & 450 \\
\hline $25 \% Q$ & 669 & 441 & 400 \\
\hline Min & 450 & 400 & 300 \\
\hline $\mathrm{p}$ [EG vs SB] & \multicolumn{2}{|c|}{0.0001} & \\
\hline$p$ [EG vs $E G / R]$ & \multicolumn{3}{|c|}{0.0002} \\
\hline $\mathrm{p}[\mathrm{SB}$ vs $\mathrm{EG} / \mathrm{R}]$ & & \multicolumn{2}{|c|}{0.2676} \\
\hline
\end{tabular}

\begin{tabular}{|c|c|c|c|}
\hline \multicolumn{4}{|c|}{$\begin{array}{l}\text { Lungencompliance niedrig, } \\
\text { UÖSD }=0.5 \mathrm{kPa}\end{array}$} \\
\hline & $E G$ & SB & $E G / R$ \\
\hline Max & 1000 & 650 & 587.5 \\
\hline $75 \% Q$ & 844 & 544 & 487.5 \\
\hline Median & 737.5 & 462.5 & 437.5 \\
\hline $25 \% Q$ & 572 & 378 & 309.5 \\
\hline Min & 375 & 200 & 200 \\
\hline$p$ [EG vs SB] & \multicolumn{2}{|c|}{0.0001} & \\
\hline $\mathrm{p}[\mathrm{EG}$ vs $\mathrm{EG} / \mathrm{R}]$ & \multicolumn{3}{|c|}{0.0001} \\
\hline$p$ [SB vs $E G / R]$ & & \multicolumn{2}{|c|}{0.1294} \\
\hline
\end{tabular}

\begin{tabular}{|c|c|c|c|}
\hline \multicolumn{4}{|c|}{$\begin{array}{l}\text { Lungencompliance niedrig, } \\
\text { UÖSD }=1.5 \mathrm{kPa}\end{array}$} \\
\hline & $E G$ & SB & $E G / R$ \\
\hline Max & 1100 & 850 & 750 \\
\hline $75 \% Q$ & 759.5 & 650 & 531.5 \\
\hline Median & 712.5 & 519 & 462.5 \\
\hline $25 \% Q$ & 662.5 & 431.5 & 344 \\
\hline Min & 437.5 & 375 & 262.5 \\
\hline$p$ [EG vs $S B]$ & \multicolumn{2}{|c|}{0.0005} & \\
\hline$p$ [EG vs $E G / R]$ & \multicolumn{3}{|c|}{0.0001} \\
\hline $\mathrm{p}$ [SB vs $\mathrm{EG} / \mathrm{R}$ ] & & \multicolumn{2}{|c|}{0.0171} \\
\hline
\end{tabular}

Signifikanzniveau $\mathrm{p}=0.016$ 
4.3.2. Magenvolumina (in $\mathrm{ml}$ )

\begin{tabular}{|c|c|c|c|}
\hline \multicolumn{4}{|c|}{$\begin{array}{l}\text { Lungencompliance hoch, } \\
\text { UÖSD }=0.5 \mathrm{kPa}\end{array}$} \\
\hline & $E G$ & SB & $E G / R$ \\
\hline Max & 375 & 300 & 175 \\
\hline $75 \% Q$ & 169 & 109.5 & 94 \\
\hline Median & 119 & 69 & 56.5 \\
\hline $25 \% Q$ & 100 & 50 & 50 \\
\hline Min & 50 & 0 & 0 \\
\hline $\mathrm{p}$ [EG vs SB] & \multicolumn{2}{|c|}{0.0010} & \\
\hline$p[E G$ vs $E G / R]$ & \multicolumn{3}{|c|}{0.0001} \\
\hline$p[S B$ vs $E G / R]$ & & \multicolumn{2}{|c|}{0.0674} \\
\hline
\end{tabular}

\begin{tabular}{|c|c|c|c|}
\hline \multicolumn{4}{|c|}{$\begin{array}{l}\text { Lungencompliance niedrig, } \\
\text { UÖSD }=0.5 \mathrm{kPa}\end{array}$} \\
\hline & $E G$ & SB & $E G / R$ \\
\hline $\operatorname{Max}$ & 450 & 375 & 200 \\
\hline $75 \% Q$ & 200 & 125 & 100 \\
\hline Median & 137.5 & 87.5 & 75 \\
\hline $25 \% Q$ & 100 & 50 & 31.5 \\
\hline Min & 0 & 0 & 0 \\
\hline$p$ [EG vs SB] & \multicolumn{2}{|c|}{0.0488} & \\
\hline $\mathrm{p}$ [EG vs $E G / R]$ & \multicolumn{3}{|c|}{0.0006} \\
\hline$p$ [SB vs $E G / R]$ & & \multicolumn{2}{|c|}{0.0674} \\
\hline
\end{tabular}

\begin{tabular}{|c|c|c|c|}
\hline \multicolumn{4}{|c|}{$\begin{array}{l}\text { Lungencompliance hoch, } \\
\text { UÖSD }=1.5 \mathrm{kPa}\end{array}$} \\
\hline & $E G$ & SB & $E G / R$ \\
\hline Max & 175 & 25 & 37.5 \\
\hline $75 \% Q$ & 72 & 0 & 25 \\
\hline Median & 50 & 0 & 0 \\
\hline $25 \% Q$ & 6.5 & 0 & 0 \\
\hline Min & 0 & 0 & 0 \\
\hline$p$ [EG vs $S B]$ & \multicolumn{2}{|c|}{0.0020} & \\
\hline $\mathrm{p}[\mathrm{EG}$ vs $\mathrm{EG} / \mathrm{R}]$ & \multicolumn{3}{|c|}{0.0059} \\
\hline $\mathrm{p}[\mathrm{SB}$ vs $\mathrm{EG} / \mathrm{R}]$ & & \multicolumn{2}{|c|}{0.3125} \\
\hline
\end{tabular}

\begin{tabular}{|c|c|c|c|}
\hline \multicolumn{4}{|c|}{$\begin{array}{l}\text { Lungencompliance niedrig, } \\
\text { UÖSD }=1.5 \mathrm{kPa}\end{array}$} \\
\hline & $E G$ & SB & $E G / R$ \\
\hline Max & 200 & 100 & 50 \\
\hline $75 \% Q$ & 75 & 0 & 0 \\
\hline Median & 50 & 0 & 0 \\
\hline $25 \% Q$ & 28 & 0 & 0 \\
\hline Min & 0 & 0 & 0 \\
\hline $\mathrm{p}$ [EG vs $\mathrm{SB}]$ & \multicolumn{2}{|c|}{0.0010} & \\
\hline $\mathrm{p}[\mathrm{EG}$ vs $\mathrm{EG} / \mathrm{R}]$ & \multicolumn{3}{|c|}{0.0010} \\
\hline $\mathrm{p}$ [SB vs $E G / R]$ & & \multicolumn{2}{|c|}{1.0000} \\
\hline
\end{tabular}

\section{Signifikanzniveau $p=0.016$}




\section{Diskussion}

\subsection{Lungenvolumina}

Laut dem ERC (European Resuscitation Council) sollte das Tidalvolumen bei der kardiopulmonalen Reanimation zwischen $500 \mathrm{ml}$ und $600 \mathrm{ml}(6-7 \mathrm{ml} / \mathrm{kg}$ Körpergewicht) betragen. Als Referenz einer suffizienten Beatmung dient bei dem Patienten eine sichtbare Erhebung der Brust (37). Alle drei Testsysteme (EG, SB und EG/R) erlaubten den Probanden, Atemzugsvolumen in dieser Grösse zu erzeugen. Diese Vorlage wurde mit allen drei Testsystemen (EG, SB und EG/R) erreicht. Dabei hat es sich gezeigt, dass unabhängig von Lungencompliance und UÖSD bei allen Probanden mit EG statistisch signifikant grössere Lungenvolumina erzielt werden konnten als mit SB oder EG/R (bis zu $1100 \mathrm{ml}$ bei niedriger Compliance und hohem UÖSD). Die Medianwerte betrugen: 662.5ml (Compliance $700 \mathrm{ml} / \mathrm{kPa}$, UÖSD 0.5kPa), 700ml (Compliance $700 \mathrm{ml} / \mathrm{kPa}$, UÖSD $1.5 \mathrm{kPa}$ ), $737.5 \mathrm{ml}$ (Compliance 500ml/kPa, UÖSD 0.5kPa) und 712.5ml (Compliance 500ml/kPa, UÖSD 1.5kPa).

Vergleichbar hohe Atemzugvolumina wurden bei Verwendung von SB und EG/R gemessen. Medianwerte von SB: $462.5 \mathrm{ml}$ (Compliance $700 \mathrm{ml} / \mathrm{kPa}$, UÖSD $0.5 \mathrm{kPa}$ ), 475ml (Compliance $700 \mathrm{ml} / \mathrm{kPa}$, UÖSD $1.5 \mathrm{kPa}$ ), $462.5 \mathrm{ml}$ (Compliance $500 \mathrm{ml} / \mathrm{kPa}$, UÖSD $0.5 \mathrm{kPa}$ ) und $518.75 \mathrm{ml}$ (Compliance $500 \mathrm{ml} / \mathrm{kPa}$, UÖSD 1.5kPa). Die niedrigsten Volumina wurden mit dem EG/R erzeugt. Medianwerte von EG/R: 425ml (Compliance $700 \mathrm{ml} / \mathrm{kPa}$, UÖSD 0.5kPa), 450ml (Compliance $700 \mathrm{ml} / \mathrm{kPa}$, UÖSD $1.5 \mathrm{kPa}$ ), $437.5 \mathrm{ml}$ (Compliance $500 \mathrm{ml} / \mathrm{kPa}$, UÖSD $0.5 \mathrm{kPa}$ ) und $462.5 \mathrm{ml}$ (Compliance 500ml/kPa, UÖSD 1.5kPa).

Zusammenfassend kann man hervorheben, dass mit allen drei Systemen eine suffiziente Lungenbeatmung erzielt werden kann. Bei Verwendung von EG ist sie im Vergleich zu SB und EG/R jedoch etwas höher. Beschränkt man sich also nur auf den Parameter des suffizienten Tidalvolumens, so können alle Systeme bei Reanimationssituationen in der zahnärztlichen Praxis verwendet werden.

\subsection{Magenblähung}

Bei 8 von 12 Testanordnungen kam es zu Einströmen von Luft in den Magen. Dabei wurden mit EG wiederum die grössten Volumina erzielt. Die Magenblähung war also bei Verwendung von EG grösser als bei Verwendung der anderen Beutel und konnte selbst bei hohem UÖSD nicht vermieden werden. Auch bei $S B$ und EG/R kam es unter gewissen Bedingungen zu einer Magenblähung. Die Medianwerte betrugen bis zu $87.5 \mathrm{ml}$ bei Beatmung mit SB bei tiefer Lungencompliance und tiefem 
UÖSD. Nur wenn UÖSD auf $1.5 \mathrm{kPa}$ angehoben wurde, konnte eine Magenblähung sowohl bei niedriger als auch bei hoher Lungencompliance bei Verwendung von SB und EG/R vermieden werden. Es hat sich also gezeigt, dass nur UÖSD bestimmend ist, ob Luft in den Magen einströmt oder nicht. Nach einem Druckabfall von $1.5 \mathrm{kPa}$ auf $0.5 \mathrm{kPa}$ kam es nämlich bei allen Beatmungssystemen unabhängig von der Lungencompliance zu einer Magenblähung.

Im Worst-Case-Szenario, bei einer Lungencompliance von 500ml/kPa und einem Sphinkterdruck von $0.5 \mathrm{kPa}$, führte die Beatmung mit Hilfe von $\mathrm{EG} / \mathrm{R}$ in mehr als $28 \%$ der Testläufe zu suffizienten Tidalvolumina. Dabei kam es zu einer signifikant kleineren Magenblähung im Vergleich zu EG. Die Verwendung von SB führte in mehr als $42 \%$ der Testläufe zu suffizienten Tidalvolumina. Die Magenblähung war vergleichbar mit derjenigen von EG. In allen anderen Versuchsanordnungen erzeugten sowohl SB als auch EG/R suffiziente Tidalvolumina. Die Magenblähung war dabei jedoch signifikant kleiner als bei Verwendung von $E G$. SB und $E G / R$ scheinen deshalb nützliche Beatmungshilfsmittel bei Reanimationssituationen für sporadisch geschultes Personal einer zahnärztlichen Praxis zu sein.

Trotzdem konnte eine Magenblähung im schlimmsten Fall der Fälle (Lungencompliance 500ml/kPa, UÖSD $0.5 \mathrm{kPa}$ ) weder bei $\mathrm{SB}$ noch bei $\mathrm{EG} / \mathrm{R}$ vollständig vermieden werden. Diese Situation widrespiegelt eine reale Reanimationssituation in einer zahnärztlichen Praxis, da es nach einem Herzkreislaufstillstand zu einem graduellen Druckabfall des unteren Ösophagussphinkters von $1.5 \mathrm{kPa}$ bis zu $0.5 \mathrm{kPa}$ kommt 20, 22. Somit lässt sich auch mit diesen beiden Beatmungsbeuteln eine Magenblähung kaum unterbinden, sobald der UÖSD tiefer wird.

\section{3. Vergleich SB und EG/R}

SB scheint gegenüber EG/R gewisse technische Vorteile zu haben. Falls der Gasfluss zu hoch ist erscheint in einem Fenster am Hals von SB ein roter Indikator. Ausserdem nimmt der Widerstand bei zu festem Zusammendrücken zu. Dies sind zwei Faktoren, die die beatmende Person vor einem zu hohen Atemwegsspitzendruck warnen. Die Beatmung kann somit angepasst werden.

Ein solches Warnsystem fehlt bei EG/R. Die Person am Beatmungsbeutel kann nur visuell kontrollieren, ob und wie viel Luft in das Reservoir entweicht, also wie stark sich das Reservoir aufbläht. Die manuelle Kontrolle durch Zunahme des Widerstandes fällt weg.

Trotz dieser zwei Warnvorrichtungen konnte bei Verwendung von SB in unseren Testläufen kein Lerneffekt erkannt werden. Die Probanden behielten die Beatmung von Beginn zu Ende hin auf dem 
gleichen Level aufrecht, das heisst, dass sie die Beatmung nicht verringerten, wenn der Widerstand zunahm oder der rote Indikator erschien. Einige verstärkten sogar den Druck als Folge der Zunahme im Widerstand. Wir vermuten, dass eine Vorinformation über diese zwei Faktoren die Probanden deutlich beeinflusst hätten, die resultierenden Volumina aber nicht statistisch signifikant verändert hätten.

De Regge et al. untersuchten die Unterschiede in Bezug auf die Beibehaltung der Ventilationsfähigkeiten zwischen einem SMART BAG® und einem gewöhnlichen Beatmungsbeutel. Unmittelbar nach dem Training führten viele Krankenschwestern die Beatmung mit einem niedrigen Tidalvolumen durch. Nach drei und sechs Monaten konnte eine Erhöhung der inspiratorischen Zeit mit SB beobachtet werden. Sechs Monate nach dem Training erzeugten sowohl SB als auch der gewöhnliche Beatmungsbeutel vergrösserte Atemwegsspitzendrucke, welche in der Gruppe der gewöhnlichen Beatmungsbeutel eine Magenblähung verursachten. In der Gruppe von SB kam es jedoch zu keiner Magenblähung. Somit scheint SB fähig zu sein, den Rückgang der Ventilationsfähigkeit im Laufe der Zeit kompensieren zu können 38.

\subsection{Material und Methode}

Ausgehend von einem Probandengut von maximal 44 Personen scheint die Beteiligung von 14 Zahnärzten klein. Ausserdem konnten grosse interindividuelle Unterschiede in der Höhe der Volumina gemessen werden. Wir empfehlen deshalb, dass man unsere Resultate in weiteren Studien mit einer grösseren Probandengruppe verifizieren sollte.

Der Versuchsaufbau widerspiegelt keine Wiederbelebungssituation bei einem realen Patienten. Die Beatmungsbeutel waren direkt an die Lungen angeschlossen und stellten somit ein luftdicht verschlossenes System dar. Laienhelfer haben jedoch oft mit einer undichten Maske zu kämpfen, was zu kleineren Tidalvolumen und einer verminderten Magenblähung führen würde. Gewöhnlich wird eine Hand benötigt, um die Maske möglichst dicht an das Gesicht des Patienten zu adaptieren und die andere Hand wird benötigt um die Beatmung durchzuführen. Da unsere Probanden beide Hände zur Beatmung benutzen konnten, wurde der Beatmungsbeutel eventuell stärker komprimiert als es in einer realen CPR Situation der Fall sein kann.

Da UÖSD durch einen Wasserspiegel simuliert wurde, gab es keine Magencompliance. Dieser Versuchsaufbau könnte daher Magenblähung oder Tidalvolumen begünstigt oder herabgesetzt haben. 
Ausserdem könnte die Beatmungsleistung durch das Hören des Lufteinstromes in das Wasser beeinflusst worden sein.

\subsection{Schlussfolgerungen}

Ausreichende Tidalvolumina konnten durch Verwendung aller drei Testvorrichtungen erzeugt werden. Somit können alle Systeme in der zahnärztlichen Praxis verwendet werden. Verglichen mit SB und $E G / R$ führt die Beatmung mit Hilfe von EG zu grösseren Atemzugsvolumina. Die erste Hypothese, dass es zwischen den drei Testapparaturen keine Unterschiede in der Grösse der Atemzugvolumen gibt, konnte somit widerlegt werden.

SB und EG/R scheinen im Vergleich zu EG sicherer zu sein, da die Magenblähung mit diesen beiden Apparaturen deutlich kleiner war (abgesehen vom Worst-Case-Szenario, wo es mit SB zu einer vergleichbaren Magenblähung kam). Die zweite Hypothese, dass die Beatmungsleistung bei Gebrauch von EG/R gleichwertig oder vergleichsweise gut ist wie bei Gebrauch von SB, konnte bestätigt werden. Beide Geräte können somit EG vorgezogen werden.

Die Kombination eines gewöhnlichen Easy Grip $\AA^{\circledR}$ Beatmungsbeutel mit einem vorgeschalteten Atembeutel scheint einen ähnlichen Schutz vor dem Risiko der Magenblähung zu liefern wie der SMART BAG®. 


\section{Literaturverzeichnis}

1. Gonzaga HF, Buso L, Jorge MA, Gonzaga LH, Chaves MD, Almeida OP. Evaluation of knowledge and experience of dentists of Sao Paulo State, Brazil about cardiopulmonary resuscitation. Brazilian dental journal. 2003;14(3):220-2. Epub 2004/04/02.

2. Zipes DP, Wellens HJ. Sudden cardiac death. Circulation. 1998;98(21):2334-51. Epub $1998 / 11 / 24$.

3. Sans S, Kesteloot H, Kromhout D. The burden of cardiovascular diseases mortality in Europe. Task Force of the European Society of Cardiology on Cardiovascular Mortality and Morbidity Statistics in Europe. European heart journal. 1997;18(12):1231-48. Epub 1998/02/03.

4. Atwood C, Eisenberg MS, Herlitz J, Rea TD. Incidence of EMS-treated out-of-hospital cardiac arrest in Europe. Resuscitation. 2005;67(1):75-80. Epub 2005/10/04.

5. Koster RW, Baubin MA, Bossaert LL, Caballero A, Cassan P, Castren M, et al. European Resuscitation Council Guidelines for Resuscitation 2010 Section 2. Adult basic life support and use of automated external defibrillators. Resuscitation. 2010;81(10):1277-92. Epub 2010/10/20.

6. Haissaguerre M, Derval N, Sacher F, Jesel L, Deisenhofer I, de Roy L, et al. Sudden cardiac arrest associated with early repolarization. The New England journal of medicine. 2008;358(19):2016-23. Epub 2008/05/09.

7. Herlitz J, Bahr J, Fischer M, Kuisma M, Lexow K, Thorgeirsson G. Resuscitation in Europe: a tale of five European regions. Resuscitation. 1999;41(2):121-31. Epub 1999/09/17.

8. Katz E, Metzger JT, Jaussi A, Schlapfer J, Fromer M, Fishmann D, et al. [What do we actually know about out-of-hospital cardiac arrest?]. Revue medicale suisse. 2005;1(9):628-30, 32-3. Epub 2005/04/09. Arret cardiaque extrahospitalier: la situation actuelle.

9. Terranova P, Valli P, Severgnini B, Dell'Orto S, Enrico Maria G. Early outcomes of out-ofhospital cardiac arrest after early defibrillation: a 24 months retrospective analysis. Indian pacing and electrophysiology journal. 2006;6(4):194-201. Epub 2006/10/13.

10. De Maio VJ, Stiell IG, Wells GA, Spaite DW. Optimal defibrillation response intervals for maximum out-of-hospital cardiac arrest survival rates. Annals of emergency medicine. 2003;42(2):242-50. Epub 2003/07/29. 
11. Klingenheben T. [Resuscitation in ventricular fibrillation: what is essential?]. Herzschrittmachertherapie \& Elektrophysiologie. 2005;16(2):78-83. Epub 2005/07/06. Reanimation bei Kammerflimmern: Worauf kommt es an?

12. Valenzuela TD, Roe DJ, Cretin S, Spaite DW, Larsen MP. Estimating effectiveness of cardiac arrest interventions: a logistic regression survival model. Circulation. 1997;96(10):3308-13. Epub 1997/12/13.

13. 2005 American Heart Association Guidelines for Cardiopulmonary Resuscitation and Emergency Cardiovascular Care. Circulation. 2005;112(24 Suppl):IV1-203. Epub 2005/11/30.

14. Berg RA, Sanders AB, Kern KB, Hilwig RW, Heidenreich JW, Porter ME, et al. Adverse hemodynamic effects of interrupting chest compressions for rescue breathing during cardiopulmonary resuscitation for ventricular fibrillation cardiac arrest. Circulation. 2001;104(20):2465-70. Epub 2001/11/14.

15. Eftestol T, Sunde K, Steen PA. Effects of interrupting precordial compressions on the calculated probability of defibrillation success during out-of-hospital cardiac arrest. Circulation. 2002;105(19):2270-3. Epub 2002/05/16.

16. Dorph E, Wik L, Stromme TA, Eriksen M, Steen PA. Oxygen delivery and return of spontaneous circulation with ventilation:compression ratio 2:30 versus chest compressions only CPR in pigs. Resuscitation. 2004;60(3):309-18. Epub 2004/03/31.

17. Fisher RS, Cohen S. Disorders of the lower esophageal sphincter. Annual review of medicine. 1975;26:373-90. Epub 1975/01/01.

18. Stelzner F, Lierse W. [The angiomuscular dilation closing of the terminal esophagus]. Langenbecks Archiv fur Chirurgie. 1968;321(1):35-64. Epub 1968/01/01. Der angiomuskulare Dehnverschluss der terminalen Speiserohre.

19. Wenzel $\mathrm{VI}, \mathrm{AH}$. The current status of ventilation strategies during cardiopulmonary resuscitation. Current opinion in critical care. 1997;3:206-13.

20. Bowman FP, Menegazzi JJ, Check BD, Duckett TM. Lower esophageal sphincter pressure during prolonged cardiac arrest and resuscitation. Annals of emergency medicine. 1995;26(2):216-9. Epub 1995/08/01.

21. Melker RJ. Alternative methods of ventilation during respiratory and cardiac arrest. Circulation. 1986;74(6 Pt 2):IV63-5. Epub 1986/12/01. 
22. Gabrielli A, Wenzel V, Layon AJ, von Goedecke A, Verne NG, Idris AH. Lower esophageal sphincter pressure measurement during cardiac arrest in humans: potential implications for ventilation of the unprotected airway. Anesthesiology. 2005;103(4):897-9. Epub 2005/09/30.

23. Spence AA, Moir DD, Finlay WE. Observations on intragastric pressure. Anaesthesia. 1967;22(2):249-56. Epub 1967/04/01.

24. Ruben H, Knudsen EJ, Carugati G. Gastric inflation in relation to airway pressure. Acta anaesthesiologica Scandinavica. 1961;5:107-14. Epub 1961/01/01.

25. Wenzel V, Idris AH, Banner MJ, Kubilis PS, Band R, Williams JL, Jr., et al. Respiratory system compliance decreases after cardiopulmonary resuscitation and stomach inflation: impact of large and small tidal volumes on calculated peak airway pressure. Resuscitation. 1998;38(2):113-8. Epub 1998/12/24.

26. Morton HJ, Wylie WD. Anaesthetic deaths due to regurgitation or vomiting. Anaesthesia. 1951;6(4):190-201; passim. Epub 1951/10/01.

27. Lawes EG, Baskett PJ. Pulmonary aspiration during unsuccessful cardiopulmonary resuscitation. Intensive care medicine. 1987;13(6):379-82. Epub 1987/01/01.

28. Girdler NM, Smith DG. Prevalence of emergency events in British dental practice and emergency management skills of British dentists. Resuscitation. 1999;41(2):159-67. Epub 1999/09/17.

29. Hunter PL. Cardiac arrest in the dental surgery. British dental journal. 1991;170(8):284. Epub 1991/04/20.

30. Brahms D. Death in dentist's chair. Lancet. 1989(2):991-2.

31. Muller MP, Hansel M, Stehr SN, Weber S, Koch T. A state-wide survey of medical emergency management in dental practices: incidence of emergencies and training experience. Emergency medicine journal : EMJ. 2008;25(5):296-300. Epub 2008/04/25.

32. Chapman PJ. A questionnaire survey of dentists regarding knowledge and perceived competence in resuscitation and occurrence of resuscitation emergencies. Australian dental journal. 1995;40(2):98-103. Epub 1995/04/01.

33. Resuscitation from cardiopulmonary arrest. Training and organization. A report of the Royal College of Physicians. Journal of the Royal College of Physicians of London. 1987;21(3):17582. Epub 1987/07/01. 
34. Skinner DV, Camm AJ, Miles S. Cardiopulmonary resuscitation skills of preregistration house officers. Br Med J (Clin Res Ed). 1985;290(6481):1549-50. Epub 1985/05/25.

35. Broadbent JM, Thomson WM. The readiness of New Zealand general dental practitioners for medical emergencies. The New Zealand dental journal. 2001;97(429):82-6. Epub 2001/11/07.

36. Mutzbauer T, Tabacek G. Device capable of reducing gastric inflation during artificial ventilation in a manikin model. The Journal of emergency medicine. 2010;38(4):428-33. Epub $2008 / 05 / 20$.

37. Handley AJ, Koster R, Monsieurs K, Perkins GD, Davies S, Bossaert L. European Resuscitation Council guidelines for resuscitation 2005. Section 2. Adult basic life support and use of automated external defibrillators. Resuscitation. 2005;67 Suppl 1:S7-23. Epub 2005/12/03.

38. De Regge M, Vogels C, Monsieurs KG, Calle PA. Retention of ventilation skills of emergency nurses after training with the SMART BAG compared to a standard bag-valve-mask. Resuscitation. 2006;68(3):379-84. Epub 2005/12/06. 